\title{
Uterine cervix metastasis from a sigmoid adenocarcinoma: a rare presentation of an uncommon tumor
}

\author{
Soufiane Berhili ${ }^{* *}$, Basma El Khannoussi ${ }^{2}$, Selma Kadiri ${ }^{1}$, Imane Mezouri ${ }^{1}$, Amine Bazine ${ }^{1}$, Asmae Touil ${ }^{1}$, Imane El Khiyat ${ }^{2}$, \\ Tayeb Kebdani ${ }^{1}$ and Noureddine Benjaafar ${ }^{1}$
}

\begin{abstract}
Metastatic carcinoma to the uterine cervix from colorectal cancer, through haematogenous or lymphatic spread, is extremely rare. We report the case of a 59 year old woman in whom cervical metastasis was diagnosed after 13 months of follow-up for a sigmoid adenocarcinoma, confirmed by immunohistochemical study, with a review of the literature. This case illustrates that abnormal gynecologic symptoms can reveal a disease progression on patients who suffer colorectal cancer.
\end{abstract}

Keywords: Uterine cervix, Metastasis, Adenocarcinoma, Sigmoid cancer, Immunohistochemistry

\section{Background}

Metastases to the female genital tract from extragenital malignancies are very uncommon, and the most common extra-genital primary sites are breast and gastrointestinal system [1,2]. Common metastatic sites of colorectal cancer include the liver, lung, lymph nodes and peritoneum, but the rarest would be the uterine cervix $[3,4]$. We report in this article a case of metastatic adenocarcinoma from a sigmoid primary to the uterine cervix, with a review of literature on uterine cervix metastasis from colorectal cancer.

\section{Case presentation}

A 59-year-old woman presented in our department with spinal cord compression at the fifth thoracic vertebrae (T5) and epiduritis, in order to receive radiotherapy on vertebral metastasis from a primary well-differentiated adenocarcinoma of the sigmoid that was diagnosed one year earlier. The patient was initially operated in September 2012 undergoing a laparotomic sigmoidectomy, post-operative cytopathology concluded in a pT4 N2 M0 stage with negative margins ( $\mathrm{R} 0$ resection). She received eight cycles of adjuvant chemotherapy (Capecitabine + Oxaliplatine) after that time at the Day-hospital of the

\footnotetext{
* Correspondence: berhili.soufiane@gmail.com

${ }^{1}$ Department of Radiotherapy, National Institute of Oncology, Ibn Sina

University Hospital, Mohamed 5 Souissi University, Rabat, Morocco

Full list of author information is available at the end of the article
}

National Institute of Oncology in Rabat, without significant adverse drug effects. During this period, physical examination and computed tomography $(\mathrm{CT})$ did not detect any local or distant relapse of the disease.

In September 2013, the patient reported whitish vaginal discharges lasting for three months earlier. In our department, physical examination did not find any neurological deficit but rather back pain lasting for 15 days due to spinal metastasis. Gynecologic examination was painful and found an easy bleeding ulcerative cervical tumor measuring approximately $6 \mathrm{~cm}$ with vaginal and bilateral parametrial involvement at rectal digital examination. Thoracic-abdominal-pelvic CT showed T5 spinal cord compression with signs of epiduritis, right pleurisy, ascites, and cervical mass extended to the corpus and measuring $68 \times 70 \mathrm{~mm}$, without bladder or rectal invasion (Figure 1). No secondary lesions were found in lung or liver. CA 19-9 serum tumor marker rate was $821 \mathrm{U} / \mathrm{ml}$ (normal $<35 \mathrm{U} / \mathrm{ml}$ ).

Cervical biopsy was performed and showed a moderately differentiated adenocarcinoma of colonic origin (Figure 2), confirmed by immunohistochemical study showing tumor cells diffusely and strongly positive for CK20 and CDX2 markers, and negative for CK7 marker (Figure 3).

10 daily fractions of decompression radiotherapy were scheduled (3 Gy per fraction), but the patient received only six fractions, she died before completing his 


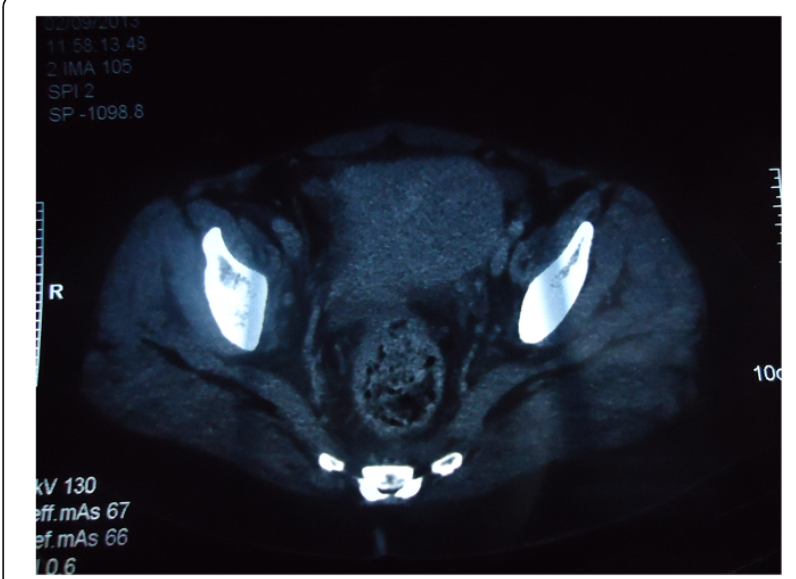

Figure 1 Computed tomography slice of the patient pelvis showing a cervico-isthmic mass.

treatment, two days after presenting a coma secondary to brain metastases, asymptomatic at the time of admission. Cervical tumor has therefore not been treated.

Involvement of the uterine cervix through direct extension of extra-genital neoplasia is frequent. However, metastatic carcinoma to the cervix through haematogenous or lymphatic spread is extremely rare $[1,3]$. Contrary to ovaries which provide a suitable environment for metastatic cells, the uterine cervix is rarely the site of metastases because of its fibrous tissue content, small size, relatively limited blood flow, and the lymphatic vessels of the pelvis all draining away from the cervix [5]. Thus, the uterus is rarely involved accounting for less than $10 \%$ of all cases of metastases to the female genital tract from extra-genital cancers (3.4\% for the uterine cervix alone) [1]. Only few cases have been reported during the past

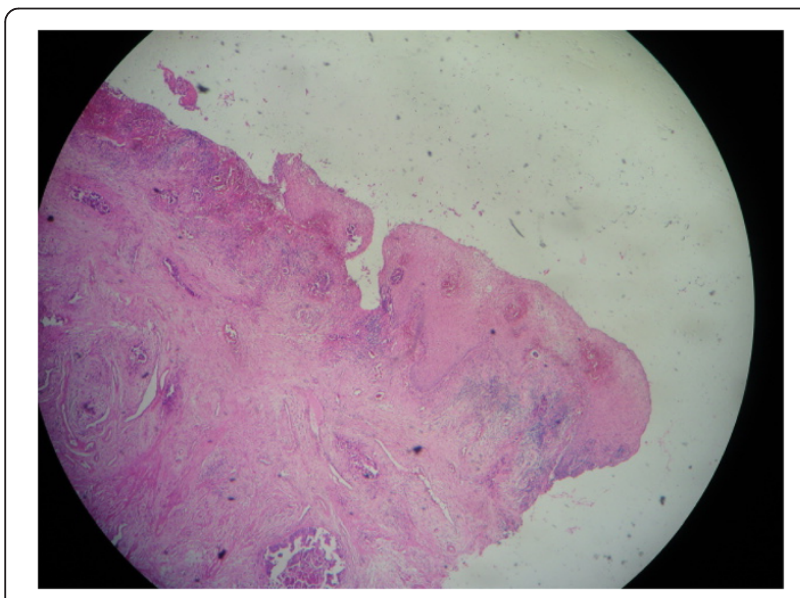

Figure 2 Cervical biopsy showing metastatic moderately differentiated adenocarcinoma cells: Ectocervical mucosa infiltrated by a carcinomatous proliferation of glands of varying size and focus of necrosis lined by atypical cells.

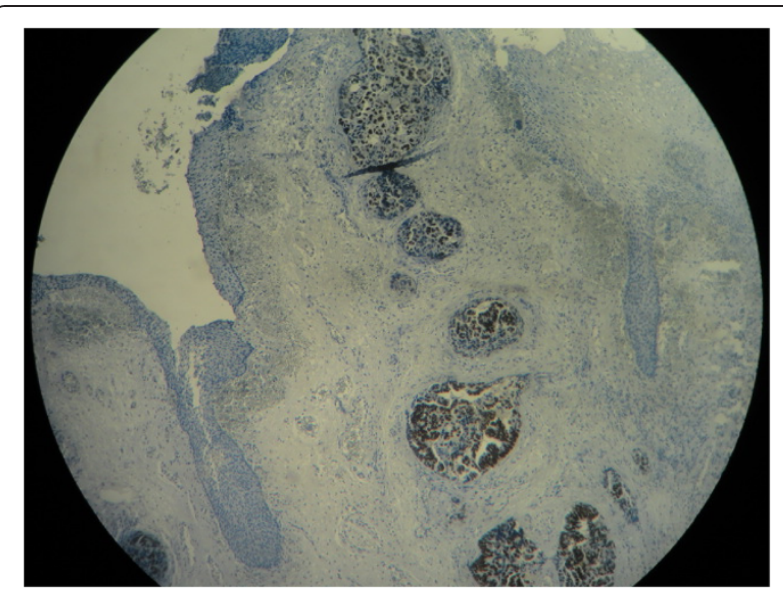

Figure 3 Immunohistochemical study of cervical tumor cells: Strong nuclear staining of tumor cells by the CDX2 marker.

several decades; the youngest patient was 17 years old [6]. These patients carry a dismal prognosis [7].

The gastrointestinal tract tumors are the most preponderant extra-genital primaries of the uterine cervix metastasis which are the stomach, colon, rectum, breast, and skin melanoma $[8,9]$.

Nakagami et al. [10], reviewed 27 cervical metastases from colorectal carcinomas, including a majority of colonic primaries. The average interval between primary carcinoma and uterine cervix metastasis appearance was 17 months (0-60 months), it was 13 months for our patient. The average survival after cervical metastasis diagnosis in Nakagami's review was 11 months.

In our case, leucorrhea appeared 3 months before the diagnosis of metastasis. Gynecological symptoms following medical history of colorectal cancer should be taken into consideration in order to refer rapidly patients for adequate treatment.

Adenocarcinoma histology type represents 0.42 to $11.7 \%$ of all cervix carcinomas [11], however the incidence of metastatic adenocarcinoma was reported to be 21.6 to $56.9 \%$ of cervical adenocarcinoma $[11,12]$.

In pathology study practice, CK7 and CK20 immunoexpresssions are commonly used in combination to evaluate immunoreactivity pattern in tumor cells, to confirm the origin of metastatic disease with unknown primary sites [13]. CK7 immunoreactivity is observed in tumors of lung, breast, upper gastrointestinal and pancreatobiliary tract, endometrium, vagina, and ovary, while tumors of colonic origin are generally non-reactive to this immunomarker. CK20 immunoexpression is detected in tumors of gastrointestinal tract and urothelium [14]. CDX2 immunoexpression has proven to be useful in establishing gastrointestinal origin in metastatic tumors, and has become a useful addition to the standard immunohistochemical stains list for carcinomas of unknown primary sites [15]. 
Thereby, in routine clinical practice and as we did, a panel combining [CK7-/CK20+/CDX2+] is used to confirm colonic primary origin $[9,13]$.

In our case, the spread of the tumor to the uterine cervix might have been caused by lymphatic and/or hematogenous pathways. Since lesion by direct extension from the primary sigmoid tumor was excluded (R0 resection was performed), and lymphatic channels of the cervix that routinely drain centrifugally, were blocked by surgery and retrograde flow might occur $[5,16,10]$.

\section{Conclusions}

In conclusion, metastases to the female genital tract from extragenital cancers are uncommon and have a poor prognosis $[9,10]$. Multidisciplinary approach including immunohistochemical study is required to diagnose these rare cases, and any gynecological symptoms following medical history of colorectal carcinoma should bear in mind metastases in order to appropriately assist these patients.

\section{Consent}

Written informed consent was obtained from the next of kin of the patient for publication of this Case report and any accompanying images. A copy of the written consent is available for review by the Editor-in-Chief of this journal.

\section{Competing interests}

The authors declare that they have no competing interests.

\section{Authors' contributions}

SB examined the patient, began cord decompressive radiotherapy, participated in the sequence alignment and drafted the manuscript. BE and IE carried out histological and immunohistochemical studies of the cervical biopsy and helped to draft the manuscript. SK, IM, AB, and AT participated in the sequence alignment. TK helped to draft the manuscript. NB coordinated between departments, revised and helped to draft the article. All authors read and approved the final manuscript.

\section{Acknowledgement}

We thank Mehdi El Amrani Omari and Adnane Mansour for their contributions in editing this English redaction.

\section{Author details \\ ${ }^{1}$ Department of Radiotherapy, National Institute of Oncology, Ibn Sina University Hospital, Mohamed 5 Souissi University, Rabat, Morocco. \\ 2Laboratory of Cytopathology, National Institute of Oncology, Ibn Sina University Hospital, Mohamed 5 Souissi University, Rabat, Morocco.}

Received: 11 March 2014 Accepted: 26 August 2014

Published: 1 December 2014

\section{References}

1. Mazur MT, Hsueh S, Gersell DG: Metastases to the female genital tract : analysis of 325 cases. Cancer 1984, 53:1978-1985.

2. Kumar NB, Hart WR: Metastases to the uterine corpus from extragenital cancers. A clinicopathologic study of 63 cases. Cancer 1982, 50:2163-2169.

3. Chereau E, Ballester M, Gonin J, Leusieur B, Darai E: Cervical metastasis from colorectal cancer. World J Oncol 2011, 2(2):83-84.

4. Cohen AM, Minsky BD, Schilsky RL: Colon Cancer. In Cancer: Principles and Practice of Oncology. 4th edition. Edited by DeVita VT Jr, Helman S, Rosenberg SA. Philadelphia: JB Lippincott Co; 1994:929-997.
5. Daw E: Extragenital adenocarcinoma metastatic to the cervix uteri. Am J Obstet Gynecol 1972, 114:1104-1105.

6. Sozen I, Small L, Kowalski M, Mayo SW, Hurwitz CA: Adenocarcinoma of the cervix metastatic from a colon primary and diagnosed from a routine pap smear in a 17-year-old woman: a case report. J Reprod Med 2005, 50:793-795.

7. Tanaka T, Kanda T, Sakaguchi S, Munakata S, Ohmichi M: Vaginal stump metastasis from sigmoid colon cancer. Acta Cytol 2012, 56:92-96.

8. Haji BE, Kapila K, Francis IM, Temmim L, Ahmed MS: Cytomorphological features of metastatic mammary lobular carcinoma in cervicovaginal smears: report of a case and review of literature. Cytopathol 2005, 16:42-48.

9. Gupta N, Dudding N, Smith JH: Cytomorphological features of extra-genital metastases in SurePath ${ }^{\text {TM }}$ cervical liquid-based cytology: a series of eight cases. Cytopathol 2013, 24:123-128.

10. Nakagami K, Takahashi T, Sugitani K, Sasaki T, Ohwada S, Morishita Y: Uterine cervix metastasis from rectal carcinoma : a case report and a review of the literature. Jpn J Clin Oncol 1999, 29:640-642.

11. William P, Abraham M: Adenocarcinoma of the cervix. Am J Obstet Gynecol 1962, 84:367-374

12. Korhonen M, Stenback F: Adenocarcinoma metastatic to the uterine cervix. Gynecol Obstet Invest 1984, 17:57-65.

13. Zulfiqar M, Liu S, Shi D, Madan S, Jacques S, King L, Shidham V, Giorgadze T: Metastatic colorectal adenocarcinoma in cervico-vaginal cytology specimens confirmed by immunocytochemical stains on liquid base specimens: two study cases with review of the literature. Cytojournal 2013, 10:9.

14. Rubin BP, Skarin AT, Pisick E, Rizk M, Salgia R: Use of cytokeratins 7 and 20 in determining the origin of metastatic carcinoma of unknown primary, with special emphasis on lung cancer. Eur J Cancer Prev 2001, 10:77-82.

15. Li MK, Folpe AL: CDX2, a new marker for adenocarcinoma of gastrointestinal origin. Adv Anat Pathol 2004, 11:101-105.

16. Lemoine NR, Hall PA: Epithelial tumors metastatic to the uterine cervix: a study of 33 cases and review of literature. Cancer 1986, 57:2002-2005.

doi:10.1186/2053-6844-1-6

Cite this article as: Berhili et al: Uterine cervix metastasis from a

sigmoid adenocarcinoma: a rare presentation of an uncommon tumor. Gynecologic Oncology Research and Practice 2014 1:6.

\section{Submit your next manuscript to BioMed Central and take full advantage of:}

- Convenient online submission

- Thorough peer review

- No space constraints or color figure charges

- Immediate publication on acceptance

- Inclusion in PubMed, CAS, Scopus and Google Scholar

- Research which is freely available for redistribution 